\title{
Inclusión educativa y parentalidad positiva: la perspectiva de niños y niñas inmigrantes venezolanos/as de primer ciclo escolar en escuelas públicas de Santiago de Chile
}

\author{
PAOLA ANDREUCCI-ANNUNZIATA*
}

Universidad Central de Chile - Chile

CLAUDIA MANSILLA-TURRA*

Universidad de Las Américas - Chile

CECILIA MONTECINO-ÁVILA**

Universidad de Las Américas- Chile

Recibido el 19-05-20; primera evaluación el 06-06-21; segunda evaluación el 28-06-21; aceptado el 09-08-21

\section{Resumen}

El estudio se inscribe en el ámbito de la inclusión educativa (IE) de niños y niñas migrantes. Se focaliza en el incremento de familias inmigrantes venezolanas en Santiago de Chile y el ingreso de sus hijos/as a primer ciclo escolar. El objetivo fue aportar voz a este grupo etario con el propósito de describir cómo la parentalidad positiva se constituye en agente protector de su IE. La investigación con aproximación cualitativa descriptiva no experimental se valió de codificación

\footnotetext{
* Doctora en Ciencias de la Educación por la Pontificia Universidad Católica de Chile (PUC) y por la Universidad Autónoma de Barcelona (UAB). Magíster en Ciencias de la Educación por la PUC. Psicóloga y Licenciada en Psicología por la Universidad de Chile. Académica e investigadora de la PUC y de la Universidad Central de Chile. Directora de la Escuela de Psicología, Universidad Central de Chile. https:// orcid.org/0000-0002-4438-0119. Correo electrónico: paola.andreucci@ucentral.cl; pmandreu@uc.cl

** Psicóloga Clínica de la Universidad de las Américas. Terapeuta EMDR en Centro Internacional de Neurociencias Aplicadas, Orientadora Familiar de la Universidad de los Lagos. Diplomada en Mediación Familiar de la Universidad Finis Terrae. Certificada en WISC-5 de la Pontificia Universidad Católica. Certificada en Trauma y Disociación. Especialización en Terapia Breve Centrada en Soluciones, Terapia Breve del Trauma y Abuso Sexual Infantil. Experiencia en el ámbito clínico en atención de niños, adolescentes y adultos. Experiencia en el ámbito educacional en el área de Orientación, Convivencia Escolar, Mediación Escolar y Programa de Integración Escolar PIE en la atención de estudiantes con NEE de tipo Permanentes y Transitorias. https://orcid.org/0000-0001-6319-8665. Correo electrónico: claudia.mansilla.turra@gmail.com

*** Psicóloga y Licenciada en Psicología por la Universidad de Las Américas, sede Providencia, Santiago, Chile. https://orcid.org/0000-0002-9066-7364. Correo electrónico: cecimontecino@gmail.com
} 
abierta guiada por los objetivos del estudio. Para ello, se aplicó un guion temático validado por tres expertos independientes, a un grupo focal de estudio de 30 estudiantes, insertos en tres escuelas públicas. Los resultados permiten concluir que las competencias parentales positivas, especialmente las de apoyo emocional, inciden positivamente en la IE.

Palabras clave: educación inclusiva; parentalidad positiva; migración; apoyo emocional.

Educational Inclusion and Positive Parenting: the perspective of Venezuelan immigrant boys and girls from the first school cycle in public schools in Santiago de Chile

\section{Abstract}

The study is part of the educational inclusion (EI) of migrant children. It focuses on the increase of Venezuelan immigrant families in Santiago de Chile and the entry of their children to the first school cycle. The objective was to provide a voice to this age group in order to describe how positive parenting becomes a protective agent of their IE. Non-experimental descriptive qualitative approach research used open coding guided by the study objectives. To do this, a thematic script, validated by three independent experts, was applied to a focus group of 30 students, inserted in three public schools. The results allow us to conclude that positive parenting skills, especially emotional support skills, have a positive impact on EI.

Keywords: inclusive education; positive parenting; migration; emotional support.

\section{Inclusáo educacional e paternidade positiva: a perspectiva de meninos e meninas imigrantes venezuelanos do primeiro ciclo escolar em escolas públicas de Santiago do Chile}

\section{Resumo}

O estudo faz parte da inclusão educacional (IE) de crianças migrantes. Ele se concentra no aumento das famílias de imigrantes venezuelanos em Santiago do Chile e na entrada de seus filhos no primeiro ciclo escolar. O objetivo era dar voz a essa faixa etária, a fim de descrever como a parentalidade positiva se torna um agente protetor do IE. A pesquisa de abordagem qualitativa descritiva, não experimental, utilizou a codificação aberta orientada pelos objetivos do estudo. Para isso, foi aplicado um roteiro temático, validado por três especialistas independentes, a um grupo focal de 30 alunos, inserido em três escolas públicas. Os resultados permitem concluir que as habilidades positivas dos pais, especialmente as habilidades de apoio emocional, têm um impacto positivo na IE.

Palavras-chave: educação inclusiva; parentalidade positiva; migração; suporte emocional. 


\section{INTRODUCCIÓN}

Los movimientos migratorios se constituyen en un fenómeno social y político universal con múltiples implicancias y desafíos de inclusión para las instituciones, las comunidades, las familias y los individuos. En la actualidad, Chile es un país que ha recibido una gran cantidad de población extranjera (Instituto Nacional de Estadísticas - INE, 2017). De acuerdo con el Departamento de Extranjería y Migración (2018) se observa que, de los permisos de permanencia definitivos entregados entre los años 2005 y 2016, la comuna de Santiago congrega un $15,7 \%$ de personas extranjeras; el $12,4 \%$ tiene entre 0 a 14 años, el $32,7 \%$ tiene entre 15 y 29 ańos y el 54,9\% tiene 30 años o más.

Según datos del Ministerio de Educación de Chile (2018), existe un aumento en la matrícula de infantes venezolanos que residen en el país, considerando el tramo que va desde educación de párvulos o preescolar, primer y segundo ciclo de enseńanza básica y enseńanza media o secundaria. Si en el año 2014 la cifra total era de 113 estudiantes, para el ańo 2016 el número había subido a 711. Específicamente, en los datos de enseñanza básica (primer y segundo ciclo), en 2014 solo existían 77 estudiantes en este tramo, en 2017 la matrícula ya había alcanzado los 2881 .

Las habilidades parentales articuladas en torno a la parentalidad positiva, por su parte, se transformaría en un agente protector de la IE, según señala la literatura reciente sobre el tema (Gomila, Pascual y Quincoces, 2018). Si se tiene en cuenta además que la población migrante «ocupa mayoritariamente condiciones sociales más vulnerables, tiene empleos más precarios, habita en viviendas peores y con mayor hacinamiento y registra peores dietas alimenticias» (Urzúa, Heredia y Caqueo-Urizar, 2016, p. 568), serían factores críticos o de riesgo que estarían incidiendo directamente no solo en la dinámica familiar y los recursos con que enfrentan el medio, sino además en la tarea de ser padres y madres.

Existen estudios que dan cuenta de la importancia que tiene la familia, su grado de involucramiento, sus pautas de interacción y de acción parental en la integración al sistema escolar de referencia, especialmente, de sus integrantes en etapa de formación inicial (Epstein et al., 2002; Epstein, 2013; Gomila, Pascual y Quincoces, 2018). Es en este contexto donde cobra importancia identificar los estilos de parentalidad positiva utilizados por padres y madres y la forma en que educan a sus hijos e hijas.

Desde esta perspectiva, los objetivos que guían el presente estudio son:

i. Identificar competencias parentales protectoras de la IE desde el discurso de niños y niñas venezolanos/as pertenecientes al primer ciclo de enseńanza primaria; 
ii. Describir condiciones de riesgo de la IE desde el discurso de nińos y niñas venezolanos/as pertenecientes al primer ciclo de enseñanza primaria.

\section{Marco teórico}

Según datos de la Organización de Naciones Unidas - ONU (2016), el número de personas que viven fuera de su país de origen alcanzó 244 millones en 2015, lo que supone un aumento de $41 \%$ con respecto al ańo 2000. «Solo en América Latina se estima un poco más de 25 millones de migrantes, una proporción superior al 13\% del total de inmigrantes internacionales» (Hernández, 2015, p. 12).

La población venezolana no ha sido la excepción. Mediante la revisión de archivos documentales y entrevistas a especialistas se ha concluido que, específicamente, la migración venezolana se relaciona con «la existencia de un grave deterioro de las condiciones de vida en Venezuela y la necesidad de un cambio profundo a nivel político, económico y social como condición necesaria para un posible retorno" (Castillo y Reguant, 2017, p. 133). La inclusión social y, específicamente, la IE de los infantes de esta población migrante venezolana no ha estado exenta de dificultades.

\subsection{Inclusión educativa (IE)}

La inclusión, como proceso educativo, supone la incorporación de la diferencia o de la alteridad a un aula educativa concebida artificialmente como homogénea con fines colaborativos, de intercambio mutuo, participación democrática y aprendizaje conjunto (Azorín, 2018; Azorín y Ainscow, 2018). La educación inclusiva es concebida como un derecho del alumnado y una condición de justicia social de instituciones gubernamentales y no gubernamentales al alero de políticas públicas tanto internacionales como locales al servicio de todos y todas (Booth y Ainscow, 2011; Echeita y Ainscow).

La IE intenta dar respuesta a la diversidad de requerimientos de aprendizaje de estudiantes cada vez más diversos, con procedencias distintas, en contextos educativos multiculturales y necesidades específicas de apoyo educativo (Charlin, Torres y Cayumán, 2016; Jiménez y Fardella, 2015; Jiménez y Montecinos, 2018). Las perspectivas de acercamiento a la IE, específicamente a la inclusión escolar, también son diversas y no están exentas de debate (Rojas y Armijo 2016). Tienden a primar enfoques práctico-pedagógicos sobre teórico-sociales (Gelber et al., 2019; Rojas, 2016) reparando, especialmente, 
sobre las prácticas pedagógicas desde los actores directamente involucrados: equipos de docentes y de directivos escolares (González-Gil, 2016).

Las sociedades de acogida, por su parte, intentan garantizar el acceso a la educación a todo el grupo de niños, niñas y jóvenes que reside en los países, aunque no necesariamente incluyan contenidos multiculturales o interculturales en sus respectivas propuestas curriculares, provocando sesgos en los contenidos evaluados durante el proceso de enseñanza-aprendizaje (Dietz, 2017; Rivera, 2020; Tubino, 2015).

En Chile, en particular, los principios inspiradores de la Ley General de Educación o Ley $\mathrm{N}^{\circ} 20.370$ (2009, p. 2) señalan que «el sistema debe reconocer y valorar al individuo en su especificidad cultural y de origen, considerando su lengua, cosmovisión e historia». Coincidimos, no obstante, en que «el sistema educativo chileno se encuentra ante un gran desafío para enfrentar la creciente diversidad estudiantil. Normativas recientes exigen a las instituciones ser más inclusivas, pero los estudios acerca del punto de partida son escasos» (Gelber et al., 2019, p. 73). Se han abordado las perspectivas de directivos y docentes, aún de este modo preliminar, sin embargo, las familias y en especial padres y madres han sido escasamente considerados tanto en las concepciones predominantes como en las prácticas implementadas al interior de las instituciones.

Este estudio pretende contribuir a dar visibilidad a las actitudes y prácticas del sistema parental y maternal de apoyo y crianza para favorecer la IE de sus hijos e hijas desde la perspectiva de los infantes.

\subsection{Parentalidad positiva}

Las actitudes de padres y madres hacia la educación y desempeño académico de sus hijos e hijas (Ames, 2012; Mayorquín y Zaldívar, 2019), así como sus estilos de educación parental o habilidades educativas relacionales a lo largo del proceso de desarrollo infanto-juvenil y sus pautas de crianza orientadas hacia el aprendizaje formal en centros educativos públicos y/o privados parecen cumplir un rol gravitante en la consecución de un proceso de inclusión educativa efectivo (Capano, González y Massonnier, 2016; Fuentes, García, Gracia y Alarcón, 2015; Suárez y Vélez, 2018). Lo anterior supone el ejercicio de buenas prácticas en parentalidad positiva y disponibilidad de recursos apropiados en padres y madres para dicho ejercicio (Máiquez et al., 2019; Sallés y Ger, 2011; Suárez-Perdomo, Byrne y Rodrigo, 2018).

Se considera que la tarea de ser padres y madres consiste principalmente en:

Velar por el bienestar físico y emocional en la nińez, pero también implica educar desde edades tempranas el tipo de actitudes y conducta consideradas 
adecuadas para el desarrollo personal y social, con el fin de que los hijos e hijas se adapten a las normas y valores de su entorno social-cultural (Calderón, 2015, p. 16).

Barudy y Dantagnan (2010, p. 34) definen la parentalidad positiva como «las capacidades prácticas que tienen las madres y los padres para cuidar, proteger y educar a sus hijos, y asegurarles un desarrollo suficientemente sano». Es en este sentido que las capacidades, competencias o habilidades parentales forman parte de una parentalidad más social que biológica y, como tal, estaría compuesta de cinco factores o necesidades que debiesen ser cubiertas para que sea considerada competente. En el detalle:

i. El aporte nutritivo, de afecto, cuidados y estimulación que refiere no solo a una alimentación con el aporte de los nutrientes necesarios para asegurar el crecimiento y prevenir la desnutrición, sino el aporte de las experiencias sensoriales, emocionales y afectivas que permitan a los infantes, por un lado, construir un apego seguro y, por otro, percibir el mundo familiar y social como un espacio confiable y no frustrante (Annemiek, Vingerhoets y Bekker, 2019; Bortolan, 2017).

ii. Los aportes educativos referentes a cuantas más experiencias de buenos tratos hayan tenido los padres y madres, más modelos y herramientas positivas y eficaces tendrán para ejercer una influencia educativa competente y moralmente positiva sobre sus hijos e hijas.

iii. Los aportes socializadores que tienen relación, por una parte, con la contribución de los padres y madres en la construcción del concepto de sí mismo e identidad de sus infantes y, por otra parte, con la facilitación de experiencias relacionales que sirvan como modelos de aprendizaje para vivir de una forma respetuosa, adaptada y armónica en la sociedad.

iv. Los aportes protectores que se aplican a dos niveles, el primero orientado a proteger a los hijos e hijas de contextos externos, familiares y sociales que puedan dañarles directamente o alterar su proceso de maduración, crecimiento y desarrollo. El segundo se orienta a protegerlos de los riesgos y peligros derivados de su propio crecimiento y desarrollo.

v. La promoción de la resiliencia que permite a los infantes la construcción de un autoconcepto y de una autoestima que los hace sentir, aún en circunstancias difíciles, que son personas dignas, valiosas y con derecho a ser respetadas y ayudadas (Gómez y Kotliarenco, 2010). 
Resituando a la familia y, especialmente a padres y madres como agentes socializadores activos para niños y niñas, implicarles en la IE y el aprendizaje académico, supondría competencias parentales, involucramiento en el proyecto educativo y compromiso educativo familiar, considerando responsabilidades compartidas entre centros educativos, familias y comunidad (Álvarez, 2019; Bravo, Salvo, Mieres, Mansilla y Hederich, 2017; Cabello y Giró, 2016).

Si bien la relación familia-escuela se constituye en un contexto idóneo para facilitar recursos, servicios y propuestas que proyecten una acción educativa exitosa, la progresión y evolución de la parentalidad positiva supone atender a las necesidades formativas de las familias en temas relacionados con el cambio de etapa evolutiva, formación a lo largo del ciclo vital, diversas dificultades relacionadas con la orientación escolar y la consideración de las necesidades y prioridades de las propias familias (Torío, Peña, García e Inda-Caro, 2019).

Lo anterior es especialmente complejo en el contexto de la migración y de las denominadas paternidades y maternidades transnacionales. Las familias y, en particular, los padres y madres experimentan una serie de reorganizaciones como estrategias para situar su convivencia diaria en una nueva sociedad. Se sostiene que estas reorganizaciones o "[...] reordenamientos familiares se acrecientan, si la que migra es la figura materna. En estos casos, las madres se enfrentan a nuevas formas de entender y ejercer la maternidad, más allá de la co-residencia y presencialidad con sus hijos» (Betancourt y Gross, 2018, p. 159). En la práctica, no obstante, tanto madres como padres se someten a la reconfiguración de sus roles, funciones, aportes y vínculos afectivos, comunicacionales y educativos incluso transfiriendo parte de estas responsabilidades a otros integrantes de la familia o de la comunidad migrante que tiende a ocupar espacios territoriales comunes (Zapata, 2009).

\section{Metodología}

El propósito del estudio fue aportar voz a niños y niñas venezolanas de primer ciclo con el objetivo de describir cómo la parentalidad positiva se constituye en agente protector de la IE. En función de lo anterior, la investigación se realizó desde una aproximación cualitativa de tipo descriptiva no experimental (Hernández-Sampieri, Fernández-Collado y Baptista-Lucio, 2014) valiéndose de codificación abierta guiada por los objetivos del estudio y la evidencia empírica levantada. Para ello, se aplicó un guion temático (Ver Cuadro 1), validado por tres expertos independientes - un metodólogo con enfoque cualitativo, un sociólogo especialista en migraciones y un psicólogo infantil- a tres grupos focales de estudio (Flick, 2007), correspondientes a treinta 
estudiantes insertos en tres escuelas públicas de tres comunas periféricas y populosas de la capital chilena. El guión temático consideró los dos objetivos y contempló dos a tres preguntas organizadas por cinco dimensiones: (1) competencias parentales vinculares o función vincular, (2) competencias parentales formativas o función formativa, (3) competencias parentales protectoras o función protectora, (4) competencias parentales reflexivas o función reflexiva, y (5) características internas/externas que favorecen o dificultan la IE. El levantamiento semántico de las categorías se realizó con el apoyo del software de análisis cualitativo Atlas Ti $7.0^{\circ}$

Como criterios de inclusión se consideraron niños y niñas venezolanas entre primero y cuarto básico (primer ciclo), de ambos sexos, con asistencia regular a sus respectivos establecimientos educacionales, cuyos padres y madres estuvieran residiendo en el país por al menos un año, con su situación legal regularizada (visa definitiva o visa democrática). Se incluyeron finalmente niños y niñas que habían ingresado a las escuelas entre 2013 y 2017, exhibían un rendimiento «bueno" (promedio calificaciones 5,5 en escala de 1,0 a $7,0)$ a objeto de controlar la variable rendimiento académico en su relación con funciones parentales (Álvarez, 2019) y que no presentaban inasistencias reiteradas durante su escolaridad (solo licencias médicas breves). Sus madres y padres presentaron un rango de edad entre 32 y 40 años, con fechas de ingreso a Chile entre 2013 y 2016 y ocupaciones de tiempo completo (secretarias, vendedores, transportistas, choferes de taxis de alquiler y estilistas).

Los resguardos éticos se abordaron desde las cartas de autorización de los establecimientos educacionales, las cartas de consentimiento informado para madres y padres y los formatos de asentimiento informado para niños y nińas. La aplicación se realizó en los recintos educativos en horario diurno, en los recreos o espacios de descanso-cambio de hora de clases. Para mayor resguardo se contó con el apoyo de las profesoras jefes quienes cautelaron de lejos (fuera de la sala en que se realizaron los focus group) el proceso ante eventuales imprevistos.

\section{Resultados}

Se seleccionan los resultados, los que se presentan mediante vińetas frente a los estímulos del guion temático del focus group, desagregados por dimensiones y objetivos. Participaron del focus group de estudiantes, un total de veinta niños y diez niñas, cinco de primero básico, cinco de segundo básico, ocho de tercero básico y doce de cuarto básico. No se logró la paridad de género dadas las condiciones o criterios de inclusión del grupo de estudio. 


\subsection{Competencias parentales vinculares o función vincular}

Respecto a la relación con sus padres y/o madres, en particular aquello que se refiere al actuar de estos cuando los/as perciben tristes, en su mayoría sostienen que el acercamiento se produce a través del diálogo y la proximidad física. Esta última resulta relevante para comprender el estilo relacional establecido con sus familias, desde su sociedad de origen hacia la de acogida.

Si, aunque sólo me preguntan cuándo estoy triste...y me abrazan. (Estudiante de 1ero. Básico)

Si, me preguntan cuándo estoy triste o desanimada. (Estudiante de 2do. Básico)

Me ayudan preguntándome quéme pasay me dan soluciones hablando. (Estudiante de 3ro. Básico)

\subsection{Competencias parentales formativas o función formativa}

Con relación a la distribución de las tareas del hogar, se hace énfasis en aquellos casos en los que se debe reiterar por parte de los padres y/o madres alguna indicación dada con antelación y valorada por ellos. La reiteración se instala como una estrategia formativa en el grupo estudiado, lo que podría responder a la adquisición de competencias o el desarrollo de funciones mentales propias de esta etapa del ciclo vital como también a un estilo formativo que responde a características culturales o de la sociedad de origen.

Estirar la sábana, cepillarme a penas me levanto, levantarme temprano para ir al colegio, cuando me porto mal en el colegio o hago algo mal me quitan las cosas que me gustan como el celular. En la mañana lo hago por mi propia cuenta, en la tarde me lo repiten muchas veces. (Estudiante 4to básico)

Tender mi cama, también barrer la casa, tender la ropa, fregar los platos cuando ellos no están (...) bueno también hacer la cola como por ejemplo la harina y el pan (cuando uno va a comprar, nos dicen "ya hijo ponte en la fila mientras compramos las cosas"). Me repiten las cosas como dos veces. (Estudiante 4to básico)

\subsection{Competencias parentales protectoras o función protectora}

Con respecto a las acciones que sus padres y/o madres les han enseñado sobre cómo actuar frente a alguna situación que eventualmente los/as ponga en riesgo y a quiénes acudir en caso de que les suceda algo, en su mayoría sostienen que es algo abordado y aprendido desde sus propios hogares y sociedades de origen.

Mi mamá me dice que no tengo que recibirle nada a extraños, que no abra la puerta a desconocidos porque si pasa algo ella no se va a dar cuenta al instante porque va 
a estar trabajando. Si me pasa algo yo acudo a algún adulto que encuentre cerca, pero que sea confiable. (Estudiante de 4to. Básico)

Mi papá se preocupa si se me acercan extraños...siempre está en posición de a la defensiva... me ha enseñado cómo defenderme y arrancar de gente desconocida que podría hacerme algo o molestarme. (Estudiante de 3ro. Básico)

\subsection{Competencias parentales reflexivas o función reflexiva}

Por otro lado, cuando son consultados/as sobre cómo perciben los conflictos entre sus madres y padres, en su mayoría sostienen que han presenciado alguna situación de esta índole y que es algo que les incomoda. Lo tienden a enfrentar desde la pasividad receptiva, aunque acusan la ambivalencia y la valencia emocional negativa que este tipo de situaciones impregnan a su autoconcepto y proceso auto reflexivo.

Siempre están discutiendo por algo, mi papá le transfirió 30 mil pesos y mi mamá le dijo que no los pudo sacar. Yo los miro, aunque no solo pelea con mi mamá, una vez estaba borracho y se peleó con su primo. Yo me siento mal, él es muy peleón, la otra vez se puso a pelear con una persona en la fila porque se coló y eso, la otra vez le dijo al chofer del Uber que se fuera más lento y el señor del Uber no sé qué le contestó, pero mi papá le dijo tonto e idiota. (Estudiante de 3ero. Básico)

"Mis papás siempre discuten cuando yo estoy descansando o viendo tele, yo no me doy cuenta, pero a veces, si me doy cuenta. Yo me siento unas veces feliz y otra triste, porque puede que estén tomando una decisión importante. (Estudiante de 1ero. Básico)

\subsection{Características que favorecen o dificultan la inclusión educativa}

El grupo de niños y niñas releva la opinión de sus padres y madres respecto de las conductas por las cuales son reconocidos/as, en especial, las actitudes que se relacionan con el buen rendimiento o el desempeño escolar, expresado en las calificaciones escolares y los incentivos materiales asociados al resultado académico obtenido. Estos últimos son especialmente relevantes en el grupo estudiado, si están asociados a afectos con valencias positivas y reconocimiento explícito tanto verbal como de aproximación física de sus adultos significativos.

Mi profesora siempre destaca que tengo notas súper buenas, que no hablo y que hago mis tareas, mi mamá me dice que me he portado muy bien y aunque no me lo diga siempre, yo sé que está contenta, a veces también me compra cosas. (Estudiante de 1ero. Básico)

A veces cuando me saco buenas notas como que me felicitan, me dan abrazos, me dan cariño y me compran cosas que me gustan. (Estudiante de 4to. Básico) 
Ahora bien, al ser consultados sobre su proceso de adaptación, reflexionan en torno a los apoyos, especialmente de su familia, docentes y algunos/as compañeros/as de curso sobre cómo han debido enfrentar distintas situaciones de discriminación en el aula o fuera de ella.

(...) en el colegio mi profesora jefa me apoya, les dice a mis compañeros que me apoyen que no es fácil comenzar de nuevo toda su vida en otro país, me sentí bien con lo que hizo la profesora, pero mis compañeros no cambiaron. (Estudiante de 2do. Básico)

No, yo siempre soy el que los invita a jugar, ellos son malos conmigo, cuando jugamos a las escondidas ellos me pegan patadas, mi mamá me dice que ellos son diferentes, que no me sienta mal por eso. (Estudiante de 3ro. Básico)

Me sentí a gusto porque me dicen que soy bueno para las calificaciones y que soy inteligente, hay una compañera que me dice que soy feo, a ella le gusta molestar, a veces me dicen que me vaya a mi país, que no me quieren acá en Chile. Los profesores me han ayudado, me recibieron bien, pude conseguir un cupo en este colegio, siento que es un buen colegio y a mis papás les parece bien y me apoyan. (Estudiante de 4to. Básico)

Cuando yo llegué a 2 do lo que más me sorprendió es que el que mejor me trataba, es el que ahora me trata más mal, en 3ero básico se portó mal conmigo, ahora está regular, me dice cejota, chocolate, oveja, me hace bullying, los profesores lo castigan, pero a él no le importa...mi papá vino a hablar con la profesora y me defendió. (Estudiante de 3ero. Básico)

Por otra parte, sobre el tiempo dedicado al estudio, es posible observar distintas visiones. Un grupo cuenta con horarios establecidos y otro solo estudia cuando debe rendir evaluaciones formales o debe realizar algún trabajo en particular. Ambos tipos de actitudes se asocian a la crianza en el seno familiar y a los acompañamientos de los padres.

Yo estudio de 18:00 a 19:00 horas... asi me enseñaron mis papás. (Estudiante de 3ero básico)

Estudio a veces cuanto tengo prueba o para algún trabajo siempre estudio, pero a veces sinceramente no estudio, siempre me ayudan mis papás cuando hay una tarea difícil...como tienen poco tiempo, los espero hasta que lleguen del trabajo. (Estudiante de 4to básico)

En relación con el apoyo de la familia en lo referente a los deberes escolares, en su mayoría el grupo sostiene que recibe algún tipo de ayuda a la hora de realizar sus tareas y/o cuando hay algo que no entienden de sus sesiones de clases. Se interpela la función formativa. 
Yo busco por internet y por los libros y cuando es muy dificil si me ayudan mis papás...si no saben les preguntan a otros de la comunidad. (Estudiante de 4to básico)

Mi papá, por ejemplo, o mi mamá, siempre me explican las cosas, mis papás me dicen "en qué te ayudo hijo" y me ayudan siempre. (Estudiante de 4to básico)

En una perspectiva complementaria, se aborda aquello que ocurre en las asignaturas en las cuales presentan mayores dificultades como en lenguaje, esto producto de los inconvenientes que tienen para entender los contenidos y en aquellas que no, como matemáticas y educación física. El rol de la familia resulta crucial en la comprensión de contenidos complejos.

Lenguaje, los profesores prácticamente nada, no nos ayudan en nada en este ramo porque siempre están cambiando de profesores...mis primos sí me ayudan...mis papás van a hablar con los profesores. (Estudiante de 3ero básico)

Lenguaje me cuesta porque ponen pruebas y no nos hacen actividades para preparar, para entender sobre el tema... mi tía me ayuda y mi mamá estudia conmigo cuando llega a casa. (Estudiante de 4to básico)

Me gustan todas, pero más Matemáticas, menos Lenguaje... a mi papá le gustan las matemáticas...trabajaba con números en Venezuela. (Estudiante de 3ero básico)

Finalmente, con relación a cómo el grupo de niños y niñas percibe la preocupación de sus familias y docentes frente a lo que puedan estar pasando incidental o sistemáticamente en el ámbito educativo en sus respectivas escuelas, en general, se releva el rol de sus padres y/o madres como cuidadores, respaldos afectivos o reforzadores fundamentales.

Mi familia si se preocupa por mis resultados y comprándome cosas para que me sienta bien y mis profesores no, nada. (Estudiante de 3ero básico)

La familia si, los profesores lo que hacen es que me dan un consejo, una orientación, que hable con el otro y mi familia siempre está pendiente de mí, que no me pase nada, que yo esté bien. Siempre se preocupan mis padres y también los de la comunidad en la que vivimos. (Estudiante de 3ero básico)

\subsection{Relación de dimensiones}

En función del análisis de contenido facilitado por el Atlas Ti $7.0^{\circledR}$, es posible apreciar el rol central como categoría aglutinante que presentan las competencias parentales positivas. Se dirigen hacia ella, en calidad de vectores, categorías de protección, formación, vínculo y reflexividad articuladas todas en torno a la búsqueda de cuidado y apoyo emocional. El apoyo se repite en los discursos con un significado polivalente de motivación, estímulo material, corrección 
de la conducta, por una parte, y cercanía emotiva o proximidad relacional, por otra.

Las variables que favorecen o dificultan la inclusión educativa están asociadas a clima emocional positivo o convivencia escolar (bullying, acoso, maltrato), por una parte, y a estrategias educativas tanto académicas como socioemocionales de apoyo implementadas por padres, madres y cuidadores principales, por otra (Ver Figura 1).

Figura 1. Relación de dimensiones y categorías de parentalidad positiva e IE

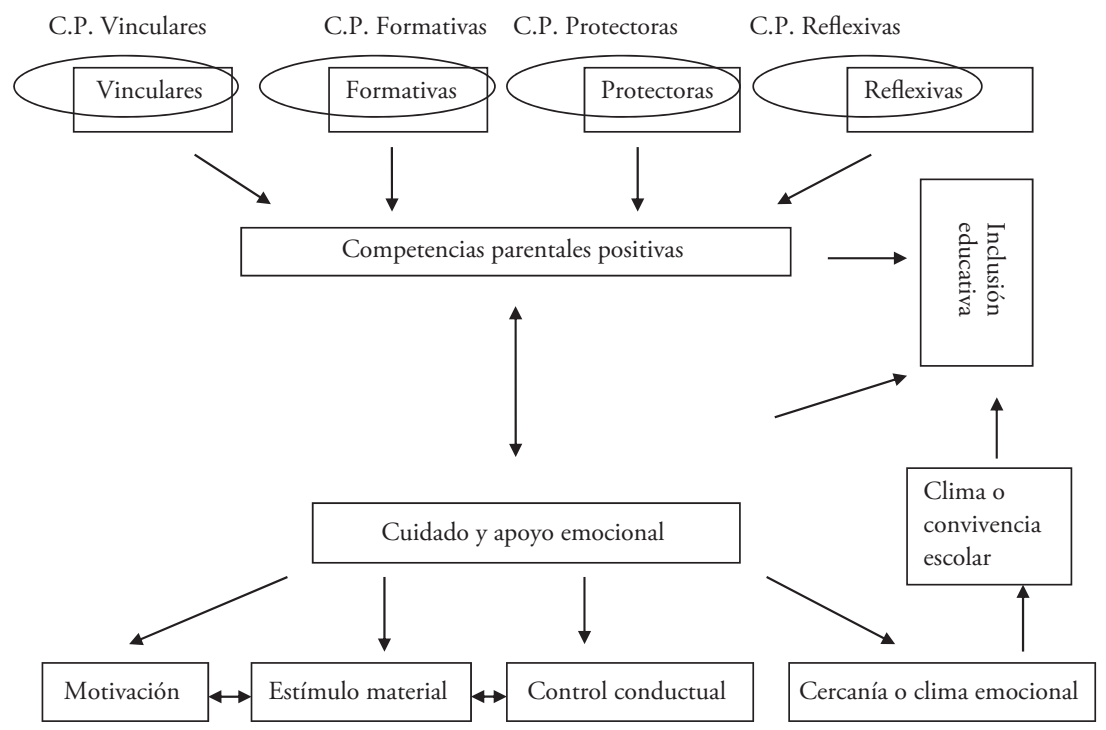

Fuente: Elaboración propia a partir de AtlasTi $7.0^{\circ}$

\section{Discusión}

Los derechos de las personas migrantes suponen velar por sus necesidades básicas y reguardar su adecuada satisfacción a lo largo de la vida (Torío et al, 2019). El derecho a educación es, sin duda, uno de los derechos fundamentales especialmente para las etapas iniciales del ciclo vital, es decir, para niños y nińas en el primer ciclo escolar. Este estudio ha intentado evidenciar la relación que existe entre la consecución de este derecho y las competencias o habilidades parentales de padres y madres a través del discurso de sus hijos e hijas. Lo anterior redundaría en una pertinente «[...] capacidad de los padres, 
madres o cuidadores/as de satisfacer las necesidades de protección, educación, cuidado y socialización a sus hijos e hijas» (Barudy y Dantagnan, 2010, p. 5).

Los resultados analizados nos permiten inferir que el ámbito de la parentalidad positiva ha sido abordado en forma competente por padres y madres, reconocido por hijos e hijas. La excepción está constituida por la dimensión o función reflexiva referida a los resguardos frente a conflictos conyugales, discusiones y peleas, en algunos casos, acrecentadas probablemente por los contextos de precariedad laboral o social que experimentan estas familias (Gaete y Mena, 2016). Las extensas jornadas de trabajo y de traslado, viviendas estrechas y acceso restringido a bienes y servicios precipitan en múltiples conflictos relacionales que afectan sus competencias reflexivas y han constituido un impedimento para ejercer de mejor forma su rol parental en esta dimensión.

Las dimensiones de protección y apoyo vincular, por su parte, aparecen como la más destacadas, siendo referidas positivamente en el grupo de nińas y nińos, tanto en el plano propioceptivo («abrazos») como verbal («felicitaciones»), con foco en la expresión de afecto frente a signos de malestar, dolor o tristeza en ellas y ellos. Siguiendo a Bortolan (2017), la experiencia afectiva es considerada singular en la medida que se vincula a estados afectivos de amor y apego, especialmente, cuando las acciones de acercamiento emocional revelan una conducta de apego con base segura (Annemiek, Vingerhoets y Bekker, 2019).

Lo anterior favorecería la adaptación emocional y académica, la asistencia escolar y la inclusión educativa según sus protagonistas, hallazgos que van en la dirección de la evidencia comparada revisada para este estudio (Bustos y Gairín, 2017). Asimismo, ponen en primer plano los lazos afectivos construidos en la infancia temprana, desde el nacimiento e incluso desde la etapa prenatal, y que pueden atribuirse a modelos de trabajo internalizados y actualizados en cuidadores/as primarios/as con ocasión de las paternidades y maternidades transnacionales (Betancourt y Gross, 2018).

El rol de la familia extendida como primos y tías aportan en dimensiones formativas y de apoyo educativo, ya que padres y madres cumplen jornadas laborales extensas lo que les restaría presencia en la función educativa y de control parental (Zapata, 2009). Lo anterior, no obstante, la crianza temprana es reconocida como un modelo o patrón orientador y resiliente por hijos e hijas que se ha internalizado y se reactualiza permanentemente, aunque el vínculo presencial con padres y/o madres se restrinja al retorno vespertino y tardío desde sus respectivos trabajos limitando la comunicación (Betancourt y Gross, 2018; Gómez y Kotliarenco, 2010). 
Por otra parte, padres y madres evidenciarían dificultad para comprender los programas educacionales chilenos, en lo referente al curriculum, lo que les resta presencia en el apoyo educativo diario, no obstante, solicitan activamente en el establecimiento educacional, a través de profesores y profesoras, apoyos diferenciales para sus hijos e hijas. Asimismo, conforman redes sociales entre ellos y con la comunidad venezolana residente y, tal como se ha visto en otras investigaciones, «[...] las asociaciones de inmigrantes juegan roles específicos y cohesivos con sus pares, incluso logran ciertas asimetrías en su favor con determinados actores de la sociedad de acogida» (Gaete y Mena, 2016, p. 149).

\section{CONClusiones}

Retomando el primer objetivo de la investigación, podemos concluir que se han logrado identificar las competencias parentales y maternales protectoras de la inclusión educativa desde el discurso de niños y nińas venezolanos/as de primer ciclo de primaria, participantes de este estudio. Los padres y madres a pesar de sus dificultades sociales, económicas, instruccionales y de acceso al conocimiento generado por la cultura de acogida, se muestran disponibles a la crianza activa de sus hijos e hijas.

La parentalidad positiva se ve desplegada tanto en aportes nutritivos, de afecto y cuidados, facilitando experiencias relacionales positivas que contribuyen a la autoestima de hijos e hijas y a una adaptación social relativamente armónica, aunque no exenta de dificultades y roces, especialmente, con los pares o compañeros/as de curso. Los padres y madres agudizan su aporte protector a través del afecto, del control en el hogar y de las indicaciones sobre defensa personal fuera del hogar. De este modo procuran promover la resiliencia (Gómez y Kotliarenco, 2010; Fuentes et al., 2015).

Fallan, no obstante, en los aportes propiamente educativos al exhibir conductas asociadas a malos tratos en el subsistema conyugal, lo que afecta su función reflexiva y limita la construcción de modelos positivos y eficaces de intercambio formativo. Lo anterior se ve graficado en este estudio por la percepción que algunos infantes tienen sobre el aporte de los docentes en su proceso de enseńanza-aprendizaje.

La IE tal y como la plantean las especificaciones técnicas propuestas por el Ministerio de Educación de Chile (2018), no se cumple del todo. La principal dificultad consiste en considerar la diferencia como una oportunidad de aprendizaje y desarrollo más que como un obstáculo y amenaza y, por ello, rechazarla de plano. En este sentido y siguiendo el segundo objetivo de este estudio, es posible describir condiciones de riesgo de la IE desde el discurso 
de niños y nińas venezolanos/as pertenecientes al primer ciclo de primaria, asociadas al acoso escolar.

$\mathrm{El}$ acoso o intimidación sistemática referida a la condición física de los y las inmigrantes venezolanos/as (color de piel, tipo de pelo, presencia física, vestuario, calzado) ha ido precipitando en estrategias de protección extrema que utilizan padres y madres para resguardarles lo más posible de la discriminación. La relación al interior de esta monoculturalidad solo diferenciada por la condición etaria, niños y niñas en este caso, invita a preguntarse por la genuina relación entre interculturalidad y educación eventualmente impactada por la violencia y la discriminación (Mondaca y Gajardo, 2015).

Finalmente, rescatamos como gran aporte de este estudio el haber otorgado voz y protagonismo a niños y nińas pequeños/as que, bajo condiciones de estricto resguardo ético, expresaron sus inquietudes en torno al proceso de adaptación e IE con el apoyo más o menos cercano de sus padres, madres y cuidadores primarios. Este protagonismo de la infancia puede verse cuestionada en transparencia y legitimidad, hasta cierto punto, por la calidad de «alteridad foránea» de las entrevistadoras al pertenecer a la cultura dominante y no a la comunidad venezolana. Este aspecto, considerado limitación del estudio, debiera mejorarse en una próxima investigación.

Por otra parte, coincidimos en que «desarrollar programas de educación parental basados en la evidencia y enmarcados en los proyectos vitales de las familias es una demanda necesaria en la actualidad» (Torío et al., 2019, p. 109). En este contexto, el trabajo en equipo entre los padres y madres, familia extendida, docentes y comunidad educativa como un todo resulta imperioso (Gelber et al., 2019).

A modo de limitaciones y proyección se espera completar este estudio con los ciclos educativos siguientes (segundo y tercero) toda vez que se evidencia cierta regresividad en conductas de mayor hostilidad en el reporte de algunos de los entrevistados. Los espacios de encuentro-desencuentro entre pares en el sistema escolar a lo largo del ciclo vital pueden requerir de mayor atención y activación de funciones parentales diferenciales, alternantes o menos presentes en etapas previas del desarrollo. En una dirección complementaria se proyecta incluir un número mayor de nińas en los grupos de estudio a objeto de visualizar eventuales diferencias con los niños en su aproximación y percepción de experiencias y habilidades parentales desde sus adultos significativos. Asimismo, considerar la inclusión de niños y niñas con desempeño académico variable a objeto de determinar eventuales influencias de las funciones parentales en la percepción de dicho rendimiento por parte del grupo de nińos (Álvarez, 2019). 
Finalmente, se proyecta abordar este fenómeno de la parentalidad positiva en un próximo estudio con la metodología de grupos triangulares conformado por padre-madre-hijo/a con el propósito de acceder a vivencias diferenciales en un escenario de mayor mediación interpersonal (Andreucci-Annunziata y Guajardo-Soto, 2016). Lo anterior podría constituirse en un aporte para la IE desde la perspectiva parental y maternal.

\section{REFERENCIAS BIBLIOGRÁFICAS}

Álvarez, L. (2019). Modelos teóricos de implicación educativa familiar: responsabilidades compartidas entre centros educativos, familias y comunidad. Aula Abierta, 48(1), 19-30. https://doi.org/10.17811/rifie.48.1.2019.19-30

Ames, P. (2012). Actitudes de madres y padres de familia hacia la educación inicial: un estudio en zonas urbanas y rurales. Educación, 21(49), 7-26. Recuperado de http://disde.minedu.gob.pe/handle/123456789/1794

Andreucci-Annunziata, P. y Guajardo-Soto, G. (2016). El potencial creativo de los grupos triangulares: una aproximación intersubjetiva y dialógica. Akademeia, 7(2), 65-76.

Annemiek K., Vingerhoets, J. J. M y Bekker, M. H. J (2019). Attachment styles and secure base priming in relation to emotional reactivity after frustration induction. Cognition and Emotion, 33(3), 428-441 https://doi.org/10.108 0/02699931.2018.1458704

Azorín C. (2018). El método de aprendizaje cooperativo y su aplicación en las aulas. Perfiles educativos, 40(161), 181-194. https://doi.org/10.22201/ iisue.24486167e.2018.161.58622

Azorín, C. y Ainscow, M. (2018). Guiding schools on their journey towards inclusion. International Journal of Inclusive Education, 3(2), 1-19. https://doi. org/10.1080/13603116.2018.1450900

Barudy, J. y Dantagnan, M. (2010). Los desafios invisibles de ser madre o padre. Manual de evaluación de las competencias y la resiliencia parental. Barcelona: Gedisa.

Betancourt, D. y Gross, A. (2018). Maternidades transnacionales. La reconfiguración de la parentalidad en familias cubanas protagonistas del proceso migratorio. Etnografias Contemporáneas, 4(6), 159-184. Recuperado de: http:// revistasacademicas.unsam.edu.ar/index.php/etnocontemp/article/view/179

Booth, T. y Ainscow, M. (2011). Guía para la educación inclusiva. Desarrollando el aprendizaje y la participación en los centros escolares (3.a ed.). Bristol: CSIE

Bortolan, A. (2017). Affectivity and moral experience: an extended phenomenological account. Phenomenology and the Cognitive Sciences, 16, 471-490. https://doi.org/10.1007/s11097-016-9468-9 
Bravo, M., Salvo, S., Mieres, M., Mansilla, J. y Hederich, C. (2017). Perfiles de desempeño académico: la importancia de las expectativas familiares. Revista de la Facultad Latinoamericana de Ciencias Sociales, 25(50), 15-35. https://doi.org/10.18504/pl2550-016-2017

Bustos, R. y Gairín, J. (2017). Adaptación académica de estudiantes migrantes en contexto de frontera. Calidad en la Educación, 3(46), 193-220. https://doi. org/10.31619/caledu.n46.7

Cabello, S. y Giró, J. (2016). La participación de las familias en la escuela: una cuestión compleja. Revista de Evaluación de Programas y Políticas Públicas, 10(7), 28-47. https://doi.org/10.5944/reppp.7.2016.16302

Calderón, D. (2015). Estrategias de intervención para suscitar competencias parentales en las familias de origen de niños y niñas vulnerados en sus derechos. (Memoria de título). Universidad de Chile, Santiago de Chile. Recuperado de http:// repositorio.uchile.cl/bitstream/handle/2250/137146/Memoria $\% 20$ de $\% 20$ T\% C3\%ADtulo\%2 0 Denise \% 20 Calder\%C3\%B 3 n. pdf?sequence $=1$ \&isAllowed $=y$

Capano, Á., González, M. L. y Massonnier, N. (2016). Estilos relacionales parentales: estudio con adolescentes y sus padres. Revista de Psicología, 34(2), 413-444. https://doi.org/10.18800/psico.201602.008

Castillo, T. y Reguant, M. (2017). Percepciones sobre la migración venezolana: causas, España como destino, expectativas de retorno. Migraciones. Publicación del Instituto Universitario de Estudios sobre Migraciones, 41, 133-163. https://doi.org/10.14422/mig.i41.y2017.006

Charlin, V., Torres, A. y Cayumán, C. (2016). Expectativas de género y logro de estudiantes en TERCE. Midevidencias, 9, 1-8. Recuperado de http://www. mideuc.cl/wp-content/uploads/2016/MidEvidencias-N9.pdf

Departamento de Extranjería y Migración. (2018). Legislación migratoria. Recuperado de: http://www.extranjeria.gob.cl/legislacion-migratoria/

Dietz, G. (2017). Interculturalidad: una aproximación antropológica. Perfiles educativos, 39(156), 192-207. https://doi.org/10.22201/iisue.24486167e. 2017.156.58293

Echeita, G. y Ainscow, M. (2011). La educación inclusiva como derecho: marco de referencia y pautas de acción para el desarrollo de una revolución pendiente. Tejeuelo, Revista de Didáctica de la Lengua y la Literatura, 12, 26-46.

Epstein, J. L. (2013). Programas efectivos de involucramiento familiar en las escuelas: estudios y prácticas. Santiago de Chile: Fundación CAP. Recuperado de: http://www.fundacioncap.cl/wpcontent/uploads/2015/09/Programas_ efectivos_de_involucramiento_familiar_en_las_escuelas-Joyce_ Epstein.pdf 
Epstein, J. L., Sanders, M. G, Simon, B. S., Salinas, K. C., Jansorn, N. R. y Van Voorhis, F. L. (2002). School, family and community partnerships: Your handbook for action. Thousand Oaks. CA: Corwin Press.

Flick, U. (2007). Introducción a la investigación cualitativa. Madrid: Morata y Fundación Paideia Galiza.

Fuentes, M. C., García, F., Gracia, E. y Alarcón, A. (2015). Los estilos parentales de socialización y el ajuste psicológico. Un estudio con adolescentes españoles. Revista Psicodidáctica, 20(1), 117-138. https://doi.org/10.1387/ RevPsicodidact.10876

Gaete, J. y Mena, L. (2016). Los roles de los actores de la sociedad de acogida en la integración de las asociaciones de inmigrantes: una aproximación desde el análisis de redes sociales. Migraciones. Publicación del Instituto Universitario de Estudios sobre Migraciones, 39, 149-181. https://doi. org/10.14422/mig.i39.y2016.006

Gelber, D., Trevińo, E., González, A., Escribano, R., Ortega, L. (2019). Del dicho al hecho: creencias y prácticas inclusivas en establecimientos y aulas escolares en Santiago. Perspectiva Educacional. Formación de Profesores, 58(3), 73-101. https://doi.org/10.4151/07189729-Vol.58-Iss.3-Art.967

Gómez, E. y Kotliarenco, M. A. (2010). Resiliencia familiar: un enfoque de investigación e intervención con familias multiproblemáticas. Revista de Psicología, 19(2), 103-131. https://doi.org/10.5354/0719-0581.2010.17112

Gomila, M.A., Pascual, B. y Quincoces, M. (2018). Family-school partnership in the Spanish education system. Journal of Education for Teaching, 44(3), 309-320. https://doi.org/10.1080/02607476.2018.1465641

González-Gil, F. (2016). Percepciones del profesorado sobre la inclusión: estudio preliminar. Revista Electrónica Interuniversitaria de Formación del Profesorado, 19(3), 11-24. https://doi.org/10.6018/reifop.19.3.219321

Hernández, C. (2015). Derechos fundamentales y migración internacional. (Tesis doctoral). Universidad de Castilla, Toledo. Recuperado de https:// ruidera.uclm.es/xmlui/bitstream/handle/10578/10131/TESIS\%20 Hern\%C3\%A1 ndez\%20Melgar.pdf?sequence $=1$ \&isAllowed $=y$

Hernández-Sampieri, R., Fernández-Collado, C. y Baptista-Lucio, P. (2014). Metodología de la Investigación. México: McGraw-Hill.

Instituto Nacional de Estadísticas - INE. (2017). Segunda entrega resultados definitivos Censo 2017. Santiago: INE. Recuperado de https://www. censo2017.cl/

Jiménez, F. y Fardella, C. (2015). Diversidad y rol de la escuela. Discursos del profesorado en contextos educativos multiculturales en clave migratoria. Revista Mexicana de Investigación Educativa, 20(65), 419-441. 
Jiménez, F. y Montecinos, C. (2018). Diversidad, modelos de gestión y formación inicial docente: Desafíos formativos desde una perspectiva de justicia social. Revista Brasileira de Educação, 23, 1-21. https://doi.org/10.1590/ s1413-24782018230005

Ley General de Educación o Ley Nº 20.370. (2009). Ministerio de Educación de Chile. Recuperado de https://www.leychile.cl/Navegar?idNorma $=1006043$

Máiquez, M., Rodrigo, M., Hidalgo, V., Amoros, P., Martínez-Gozález, R., Arranz, E., Ochaita, E., Martín-Quintana, J. y Balsells, M. (2019). Protocolo de buenas prácticas en parentalidad positiva. Su elaboración y estudio piloto. Revista INFAD de Psicología. International Journal of Developmental and Educational Psychology, 2(1), 425-436. https://doi.org/10.17060/ijodaep.2019.n1.v2.1477

Mayorquín, E. y Zaldívar, A. (2019). Participación de los padres en el rendimiento académico de alumnos de primaria. Revisión de literatura. RIDE. Revista Iberoamericana para la Investigación y el Desarrollo Educativo, 9(18), 868-896. https://doi.org/10.23913/ride.v9i18.480

Ministerio de Educación de Chile (2018). Recuperado de: https://centroestudios.mineduc.cl/wp-content/uploads/sites/100/2018/12/ANUARIOMINEDUC_VERSION-BAJA.pdf

Mondaca, C.y Gajardo, Y.(2015). Interculturalidad, migrantesyeducación. Diálogo andino, 47, 3-6. https://doi.org/10.4067/S0719-26812015000200001

Organización de Naciones Unidas - ONU (2016). ONU cifra en 244 millones los migrantes que viven fuera de su país. Recuperado de: https://news.un.org/ es/story/2016/01/1348461

Rivera, E. (2020). La interculturalidad como contenido transversal en la educación universitaria peruana. Notas reflexivas. Educación, 29(56), 211-210. https://doi.org/10.18800/educacion.2020.010

Rojas, M. T. (2016). Inclusión social en las escuelas: estudio de prácticas pedagógicas inclusivas y proyecciones para enfrentar un escenario sin copago y selección escolar (N.o 911429). Santiago: Universidad Alberto Hurtado.

Rojas, M. T. y Armijo, M. (2016). Qué es la inclusión escolar: distintas perspectivas en debate. Cuaderno de Educación, 75(2), 3-18.

Sallés, C. y Ger, S. (2011). Las competencias parentales en la familia contemporánea. Educación social: Revista de intervención socioeducativa, 49(25), 39-47.

Suárez, P. y Vélez, M. (2018). El papel de la familia en el desarrollo social del niño: una mirada desde la afectividad, la comunicación familiar y estilos de educación parental. Revista Psicoespacios, 12(20), 153-172. https://doi. org/10.25057/issn.2145-2776 
Suárez-Perdomo, A., Byrne, S. y Rodrigo, M. J. (2018). Assessing the ethical and content quality of online parenting resources. Comunicar, 26(54), 19-28. https://doi.org/10.3916/C54-2018-02

Torío, S., Peña, J. V., García, O., e Inda-Caro, M. (2019). Evolución de la Parentalidad Positiva: Estudio longitudinal de los efectos de la aplicación de un programa de educación parental. Revista Electrónica Interuniversitaria de Formación del Profesorado, 22(3), 109-126. https://doi.org/10.6018/ reifop.389621

Tubino, F. (2015). La interculturalidad en cuestión. Lima: Fondo Editorial de la PUCP.

Urzúa, A., Heredia O. y Caqueo-Urízar, A. (2016). Salud mental y estrés por aculturación en inmigrantes sudamericanos en el norte de Chile. Revista Médica de Chile, 20(144), 563-570. https://doi.org/10.4067/ S0034-98872016000500002

Zapata, A. (2009). Familia transnacional y remesas. Revista Latinoamericana de Ciencias Sociales, Niñez y Juventud, 7(2), 1749-1769. Recuperado de http://www.scielo.org.co/scielo.php?script=sci_arttext\&pid=S1692715X 2009000300024 